Résumés des conférences et travaux

\title{
Linguistique baltique et indo-européenne
}

\section{Linguistique baltique et indo-européenne}

Conférences de l'année 2014-2015

\section{Daniel Petit}

\section{OpenEdition \\ Journals}

Édition électronique

URL : https://journals.openedition.org/ashp/1892

DOI : 10.4000/ashp.1892

ISSN : 1969-6310

Éditeur

Publications de l'École Pratique des Hautes Études

Édition imprimée

Date de publication : 1 septembre 2016

Pagination : $374-378$

ISSN : 0766-0677

Référence électronique

Daniel Petit, « Linguistique baltique et indo-européenne », Annuaire de l'École pratique des hautes études (EPHE), Section des sciences historiques et philologiques [En ligne], 147 | 2016, mis en ligne le 05 octobre 2016, consulté le 06 juillet 2021. URL : http://journals.openedition.org/ashp/1892 ; DOl : https:// doi.org/10.4000/ashp.1892 


\title{
LINGUISTIQUE BALTIQUE ET INDO-EUROPÉENNE
}

\author{
Directeur d'études : M. Daniel PetiT
}

Programme de l'année 2014-2015 : I. Introduction à la syntaxe historique des langues indoeuropéennes. - II. Introduction à la linguistique historique des langues baltiques.

Le séminaire de linguistique baltique et indo-européenne a été divisé, pour l'année 2014-2015, en deux parties égales, la première consistant en une introduction à la syntaxe historique des langues indo-européennes, la seconde portant sur les conjugaisons réfléchies des langues baltiques (lituanien, letton, vieux prussien). Chacune de ces deux parties, occupant l'une des deux heures du séminaire, reflète des travaux en cours du directeur d'études et a permis d'offrir à ses auditeurs deux approches différentes de la linguistique indo-européenne, une approche plus théorique et généraliste d'une part, et une approche plus philologique et textuelle d'autre part.

Le choix d'aborder à nouveaux frais la syntaxe des langues indo-européennes résulte d'une constatation étonnante : alors qu'il existe depuis le XIX ${ }^{\mathrm{e}}$ siècle un nombre important de travaux dans ce domaine, on répète continuellement l'idée que la syntaxe est le parent pauvre des études indo-européennes. La raison en est probablement le sentiment d'un état relativement vague et imprécis de la syntaxe au sein de la grammaire comparée. En premier lieu, la syntaxe est encore trop souvent traitée par les indo-européanistes comme un appendice de la morphologie, en contradiction avec les tendances actuelles de la linguistique, qui accordent à la syntaxe une place de premier plan (parfois dans des proportions excessives). Un deuxième obstacle est le lien encore trop souvent établi entre syntaxe et stylistique, comme si la syntaxe relevait de choix individuels et, à ce titre, n'obéissait à aucune régularité. La syntaxe indo-européenne a, enfin, souffert du préjugé selon lequel il serait impossible de reconstruire la syntaxe des proto-langues : Dressler (1969), par exemple, estimait que « la syntaxe n'est pas reconstructible avec les méthodes des Néo-grammairiens ». Ces préjugés ont fait l'objet, durant le séminaire, d'une critique approfondie, visant à montrer qu'on peut appliquer la syntaxe historique à la reconstruction de l'indo-européen, avec autant de certitude, ou d'incertitude, que la phonologie ou la morphologie historiques.

Une définition générale de la syntaxe repose sur la notion de « connexion » associant un élément dominant ( «tête», anglais head) et un élément dominé (« dépendant », anglais dependent), en des termes qui se rattachent plus ou moins aux vues défendues au sein de la grammaire de dépendance. Une discussion a été menée sur les deux principales réalisations formelles de la connexion, l'accord (agreement) et la rection (government). Ce qui frappe est l'isofonctionnalité des deux principes de connexion, ce qu'on a illustré par des exemples d'équivalence entre accord et rection en indo-européen. On peut, par exemple, attribuer à l'indo-européen un modèle syntaxique permettant de coordonner un adjectif possessif (relevant de l'accord) et un

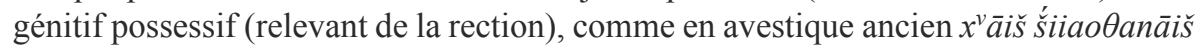


hizuuascā «par ses propres actions et celles de sa langue» (adj. poss. $x^{v} \bar{a} i \check{s}$ «ses propres » et gén. poss. hizuuas « de la langue », reliés par $-c \bar{a}$ « et »). L'équivalence fonctionnelle entre accord et rection peut également être illustrée par des exemples d'interchangeabilité paradigmatique, comme dans la coexistence d'un adjectif prédicatif accordé au nom-tête et d'un substantif prédicatif régi à l'instrumental en polonais : on jest piękny « il est beau » (accord de l'adjectif), vs on jest żotnierzem « il est soldat » (rection à l'instrumental prédicatif). Si l'on se fonde sur l'accord et la rection, c'est-à-dire exclusivement sur des indices formels, l'identification de la « tête » et du « dépendant » paraît simple, même s'il existe des cas ambigus, comme celui des « substantifs-épithètes » où chacun des deux substantifs associés peut parfois reven-

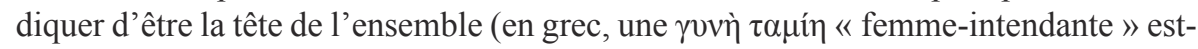
elle une femme intendante ou une intendante femme?). Un débat souvent présenté comme crucial est celle du rapport entre « tête » et « sujet » : on a tendance à identifier sujet et nominatif (ce qui laisse de côté les sujets dits «non-canoniques »), mais ce sujet est-il la tête du verbe, ou le verbe la tête du sujet? Toutes ces questions ont été traitées par l'examen d'exemples pris à des langues indo-européennes diverses; le cas du parfait transitif de l'arménien classique a par exemple donné lieu à une longue analyse. Plus globalement, on a souvent considéré comme unidirectionnel le rapport formel entre tête et dépendant, la tête imprimant son accord ou sa rection sur le dépendant, mais on a montré qu'il existait dans les langues indo-européennes des exemples de marquage morphologique de la tête (head-marking). Deux d'entre eux ont été étudiés en détail : l'ezafé iranien (cf. persan ketāb-e pedar « livre du père »), dont l'origine a été élucidée, et la suppression de l'article défini sur la tête déterminée par une tournure possessive en celtique (cf. cornique chŷ an dên « [la] maison de l'homme »).

Une autre approche de la relation tête-dépendant repose sur la notion d'autonomie syntaxique : on définit, intuitivement, la tête comme l'élément dont la suppression rend impossible l'insertion du dépendant dans l'énoncé, et inversement le dépendant comme celui dont la suppression n'a pas d'effet sur la tête. On peut montrer les limites de cette vision des choses. D'abord, il peut y avoir ellipse de la tête, comme dans le

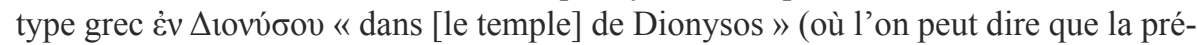
position sert de tête de substitution) et plus encore dans le type hittite $\check{s} \bar{a} u$ ataraš $«$ le sonneur de cor » (littéralement : « [celui] du sonner de cor »), type étudié récemment par I. Yakubovitch. Ensuite, on peut difficilement appliquer ce critère à ce qu'on appelle la «subordination asyndétique» (type latin : credo misericors est « je crois [qu']il est miséricordieux »), où la suppression de la tête, la proposition principale, ne suscite aucune agrammaticalité du dépendant, la proposition subordonnée.

Une dernière approche de la relation tête-dépendant porte sur le sémantisme des structures. Il peut arriver qu'une tête suscite chez son dépendant une contrainte sémantique, comme lorsqu'une préposition implique un objet animé (par ex. la préposition apudessive de l'albanais tek « chez », seulement avec animé) ou à l'inverse lorsque le sémantisme du verbe introducteur sélectionne une proposition infinitive ou participiale (par ex. gr. $\lambda \dot{\varepsilon} \gamma \omega$ « je dis »+ prop. infinitive, $v s$ ó $\tilde{\omega}$ « je vois »+ prop. participiale).

La syntaxe historique a des liens étroits avec la distinction tête-dépendant. Bon nombre des évolutions syntaxiques se ramènent à des modifications de cette relation fondamentale. On a illustré cette question par des cas de réanalyse syntaxique 
impliquant un «reparenthésage » (rebracketing). La question qui se pose est celle de savoir si la syntaxe historique porte sur des évolutions des constituants ou sur des évolutions des structures. Il existe en syntaxe des approches qui postulent une immuabilité des structures profondes, le changement syntaxique ne portant que sur la surface, ou d'autres approches, qui, à l'inverse, limitent l'objet de la syntaxe historique aux changements des structures profondes, le reste n'étant qu'épiphénomène. On a soumis à la critique ces deux approches extrêmes, qui reposent sur une vision simpliste du changement syntaxique, alors que toute évolution suppose, par nature, une interaction. Certains estiment par exemple qu'il n'y a pas de changement syntaxique entre le latin lentē «lentement» et l'espagnol lentamente «lentement», puisqu'audelà du remplacement formel, les fonctions sont restées les mêmes. On a montré que cela n'est pas le cas et que l'introduction des adverbes en *-mente dans les langues romanes a eu des effets sur les fonctions adverbiales : les adverbes en *-mente sont exclus des fonctions argumentales (esp. Anna come picante "Anne mange épicé », jamais *Anna come picantemente). Un autre exemple d'interaction entre constituants et structures est l'évolution des infinitifs latins en -re vers le statut de nom verbal dans les langues romanes; en ancien français, ce passage a pu occasionnellement être marqué formellement par l'introduction des marques flexionnelles (anc. fr. il corners « le fait de sonner le cor», avec $-s$ du cas sujet), et, en roumain, par exemple, les infinitifs peuvent être traités comme des substantifs (féminins), tout en gardant leurs propriétés verbales (vieux-roum. mărturisire-a pre Hristos «le fait de confesser le Christ », avec marquage différentiel de l'objet), ce qui n'est pas possible en français (*le lancer un javelot, vs le lancer de javelot). Un dernier exemple a été celui de l'adverbialisation des adjectifs prédicatifs en allemand moderne (er ist gut, sie ist gut, sie sind gut), dont l'origine et le développement ont été suivis depuis le vieux-haut-allemand jusqu'à nos jours.

La seconde partie de la conférence a été consacrée aux conjugaisons réfléchies des langues baltiques. Un des traits caractéristiques de ces langues est l'existence de conjugaisons associant une forme verbale et une «particule» réfléchie : lituanien praũsti « laver », vs praũsti-s « se laver»; letton mazgāt « laver », vs mazgātie-s « se laver »; vieux-prussien dātun « donner », vs dātun-si « se donner». D’emblée, on a l'impression que ce développement est une innovation aréale, dont on retrouve le principe en slave (v. slave myti «laver», vs myti se « se laver ») et en scandinave (v. isl. setja « asseoir », vs setja-sk « s'asseoir »). Mais il existe entre ces constructions des divergences importantes. Alors qu'en slave et en baltique la particule réfléchie est indifférente à la personne et se présente comme un réfléchi général (lit. prausiúo-si « je me lave», pol. myję się « je me lave »), elle a connu un marquage en personne en scandinave ancien (v. isl. finno- $m k$ « je me trouve », vs fin-zk « il se trouve », avec une première personne du sg. $-m k<m i k$ «moi », $v s$ une troisième personne du $\mathrm{sg}$. $-s k<$ $s i k$ « soi »), avant de se figer sous la forme $-s(k)$ (cf. isl. kallast « je m'appelle »). Une autre différence est qu'en baltique et en slave la valeur réfléchie prédomine encore (avec un développement marginal d'emplois passifs, plus répandu en slave qu'en baltique), tandis qu'en scandinave cette formation a fini par devenir une simple marque de passif (suédois $-s$ ). Comparer : 


\begin{tabular}{|c|c|c|c|c|c|}
\hline & $\begin{array}{l}\text { Réfléchi direct } \\
\text { (diathèse récessive) }\end{array}$ & $\begin{array}{l}\text { Réfléchi indirect } \\
\text { (bénéfactif) }\end{array}$ & Réciproque & Déponent & Passif \\
\hline Lituanien & $\begin{array}{l}\text { máudyti-s } \\
\text { «se baigner » }\end{array}$ & $\begin{array}{l}\text { pirkti-s } \\
\text { «acheter pour soi » }\end{array}$ & $\begin{array}{l}\text { bárti-s } \\
\text { «se quereller» }\end{array}$ & $\begin{array}{l}\text { džiaũgti }-s \\
\text { « se réjouir » }\end{array}$ & (rare) \\
\hline Letton & $\begin{array}{l}\text { celtie-s } \\
\text { «se lever » }\end{array}$ & $\begin{array}{l}\text { iepirktie-s } \\
\text { «faire ses courses » } \\
(\text { rare })\end{array}$ & \begin{tabular}{|l} 
pazìties \\
« se connaître »
\end{tabular} & $\begin{array}{l}\text { bitie-s } \\
\text { «craindre » }\end{array}$ & (rare) \\
\hline Vieux-prussien & $\begin{array}{l}\text { maitātun-sin } \\
\text { «se nourrir » }\end{array}$ & $\begin{array}{l}\text { enimmimai sin } \\
\text { «s'emparer de » }\end{array}$ & (pas d'exemple) & $\begin{array}{l}\text { audāst sien } \\
\text { «se passer » }\end{array}$ & $\begin{array}{l}\text { (pas } \\
\text { d'exemple) }\end{array}$ \\
\hline Russe & $\begin{array}{l}\text { myt'-sja } \\
\text { «se laver » }\end{array}$ & $\begin{array}{l}\text { stroit' }- \text { sja } \\
\text { « construire pour } \\
\text { soi » }\end{array}$ & $\begin{array}{l}\text { celovat'-sja } \\
\text { «s'embrasser » }\end{array}$ & $\begin{array}{l}\text { serdit'-sja } \\
\text { «se fâcher » }\end{array}$ & $\begin{array}{l}\text { delat'sja } \\
\text { «se faire, } \\
\text { être fait } »\end{array}$ \\
\hline Vieil-islandais & $\begin{array}{l}\text { binda-sk } \\
\text { « se lier » }\end{array}$ & $\begin{array}{l}\text { beipa-sk } \\
\text { «réclamer pour } \\
\text { soi » }\end{array}$ & $\begin{array}{l}\text { berja-sk } \\
\text { «se battre » }\end{array}$ & $\begin{array}{l}\text { fraeva-sk } \\
\text { «réussir » }\end{array}$ & $\begin{array}{l}\text { finna-sk } \\
\text { «se trouver, } \\
\text { être trouvé » }\end{array}$ \\
\hline
\end{tabular}

Les conjugaisons réfléchies des langues baltiques, slaves et scandinaves présentent de nombreuses similitudes avec la voix moyenne en indo-européen, et l'on a supposé depuis longtemps qu'elles en étaient le renouvellement historique. Les travaux sur la voix moyenne (par ex. Kemmer 1993) ont montré que la polysémie de ses formes recouvrait à peu près celle des conjugaisons réfléchies du baltique, du slave et $\mathrm{du}$ scandinave :

\begin{tabular}{|c|c|c|c|c|c|}
\hline & \begin{tabular}{|l|} 
Réfléchi direct \\
(diathèse récessive)
\end{tabular} & $\begin{array}{l}\text { Réfléchi indirect } \\
\text { (bénéfactif) }\end{array}$ & Réciproque & Déponent & Passif \\
\hline Grec ancien & 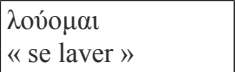 & 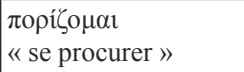 & $\begin{array}{l}\dot{\alpha} \gamma \omega v i \zeta \zeta o \mu \alpha \imath \\
\text { «se battre » }\end{array}$ & 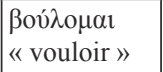 & $\begin{array}{l}\lambda \text { ov́o } \mu \alpha 1 \\
\text { « être lavé » }\end{array}$ \\
\hline Latin & $\begin{array}{l}\text { lauor } \\
\text { «se laver } »\end{array}$ & $\begin{array}{l}\text { pigneror } \\
\text { «prendre en gage» }\end{array}$ & $\begin{array}{l}\text { complector } \\
\text { «s'embrasser } »\end{array}$ & $\begin{array}{l}\text { loquor } \\
\text { «parler» }\end{array}$ & $\begin{array}{l}\text { lauor } \\
\text { « être lavé » }\end{array}$ \\
\hline Sanskrit & $\begin{array}{l}\text { vártate } \\
\text { «se tourner » }\end{array}$ & $\begin{array}{l}\text { yájate } \\
\text { «sacrifier pour soi » }\end{array}$ & $\begin{array}{l}\text { yúdhyate } \\
\text { «se battre » }\end{array}$ & \begin{tabular}{|l|} 
jușáte \\
«se réjouir »
\end{tabular} & $\begin{array}{l}\text { formation } \\
\text { spécifique : } \\
\text { budhyáte } \\
\text { « être éveillé » }\end{array}$ \\
\hline
\end{tabular}

Plus précisément encore, certains « déponents » des langues à voix moyenne ont des correspondants eux aussi « déponents » dans les langues à conjugaison réfléchie : lituanien bósti-s «éprouver du dégoût», vs sanskrit bhắdhate "faire pression »; lituanien bijóti-s, vieux slave bojati sę « craindre », vs sanskrit bháyate « avoir peur »; lituanien pasigèsti « désirer, regretter», vs grec $\theta \dot{\varepsilon} \sigma \sigma \alpha \sigma \theta \alpha$; letton smiêtiês, vieux slave smijati se « sourire, rire », vs sanskrit smáyate. Il existe cependant des divergences : lituanien bégti « courir » (non-réfléchi), vs grec $\varphi \varepsilon ́ \beta \beta \mu \alpha \imath ~ «$ fuir » (moyen);

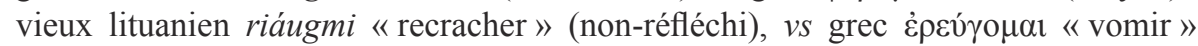
(moyen, mais le latin a èrūgō, actif); lituanien mirti «mourir» (non-réfléchi), vs sanskrit mriyáte, latin morior (moyen). Ce qu'on observe, en revanche, c'est une large convergence balto-slave : lituanien bijóti-s et vieux slave bojati sę « craindre » (mais le vieux prussien a biātwei, non-réfléchi); lituanien mélsti-s et vieux slave moliti sę

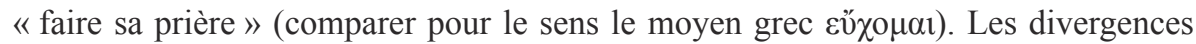


entre le baltique et le slave sont rares : lituanien šviẽsti ou švitéti «briller » (nonréfléchi), vs vieux slave svitěti sę « briller» (réfléchi).

Une seconde étape de la réflexion a conduit à s'interroger sur les formes des pronoms personnels et à leur rapport avec la particule réfléchie. En lituanien, la particule réfléchie -si prolonge un ancien datif, auquel répondent $m i$ et $t i$ pour la première et la deuxième personne du singulier; en slave, en revanche, il faut partir d'un accusatif se $(<* s \bar{e}-m)$. Les données du prussien sont compliquées : la particule réfléchie -sin paraît refléter un accusatif (cf. sien, forme tonique, parallèle à mien et tien), mais on a des exemples de -si, qui pourrait refléter l'ancien datif (à moins qu'elle ne représente l'ancien accusatif écrit/si/ pour/sî/, abréviation de /sin/); il n'y a en tout cas aucune corrélation entre la graphie -si et une valeur de réfléchi indirect. La flexion des pronoms personnels en balto-slave a été présentée en détail, afin de déterminer la position de ces formes enclitiques par rapport aux formes toniques et leur survie dans les langues modernes. Pour finir, on a discuté des règles de position qui les régissent : position postverbale dans la majorité des langues (type russe on moet-sja « il se lave »), position de Wackernagel encore en polonais (on się myje « il se lave »), voire interposition en lituanien dans les verbes préverbés (lit. praũsia-si « il se lave», mais pa-si-praũsia « il se lave un peu », ne-si-praũsia «il ne se lave pas »). Les particularités formelles des conjugaisons réfléchies seront étudiées l'année prochaine. 